Supporting the social triad

Supporting the social triad. A Commentary on Keeping Culture in Mind: A Systematic Review and Initial Conceptualization of Mentalizing from a Cross-Cultural Perspective

Peter Fonagy, Research Department of Clinical, Educational and Health Psychology, University College London, UK; Anna Freud National Centre for Children and Families, London, UK.

E-mail: p.fonagy@ucl.ac.uk

Chloe Campbell, Research Department of Clinical, Educational and Health Psychology, University College London, UK; Anna Freud National Centre for Children and Families, London, UK.

E-mail: c.campbell@ucl.ac.uk

Correspondence to Professor Fonagy 


\section{Supporting the social triad. A Commentary on Keeping Culture in Mind: A Systematic Review and Initial Conceptualization of Mentalizing from a Cross-Cultural Perspective}

We read Aival et al.'s systematic review (Aival, Rothschild-Yakar, \& Kurman, 2019) on mentalizing from a cross-cultural perspective with great interest and appreciation of the authors' thoughtful and thorough approach. We particularly appreciate Aival et al'.s clear statement of the triad of factors key to the comprehensive understanding of this question: attachment, mentalizing and epistemic trust (the social learning triad). The common conflation of these subdomains is understandable given their interdependence, potential mutual facilitation and hindering. Thus, however we operationalize mentalizing we have to assume that historically it represents the sequelae of attachment history and the confounding capacity to create productive socially and cognitively informative interpersonal relationships. As Aival et al. helpfully identify, the likelihood of generating or receiving socially informative communication from a cultural system depends on the extent to which the individual has a realistic expectation of feeling recognised as an agent, the primary cue to which is the experience of being mentalized.

In this commentary we would like to underscore two aspects of Aival et al.'s exceptionally clear and productive review. The first concerns the broadening of current views of attachment associated with cultural variability in mentalizing identified in this systematic review. The second entails how epistemic trust may interface with these cultural dimensions to generate vulnerability to subsequent 
Supporting the social triad

adversity, which may account for the observed association between mental disorder and mentalizing reported in this meta-analysis.

The authors relate evidence that indicates substantial cultural variations in the four overlapping empirical domains underpinning mentalizing: theory of mind, empathy, perspective taking, and alexithymia. It seems that people in individualistic cultures tend to focus more on mental states within the self than on the mental states of others, and there are variations in the way in which people from different cultures tend to perceive mental states. The review indicates a cultural effect on mentalizing which coincides with what we have suggested as the implicit/explicit polarity of mentalization. It seems that cultural variations are more significant in the effortful and verbal aspect of people's mindreading - in Kahneman's terms, the slow rather than fast cognitive pathway (Kahneman, 2011). Cultural influence is less prominent in the automatic, fast and less verbally weighted ability to implicitly perceive mental states. The review attributes differences to a) difference in lexical explicitness and b) grammaticalised epistemic concepts. The study also raises the potential influence of value preference based on studies that go beyond the dichotomous comparison between individualistic and collectivist cultures. Aival et al. point to, but do not fully elaborate on, known differences in parenting practices and characteristics across cultural groups.

We have previously drawn attention to the anthropological critiques of attachment theory rooted in the profound and overwhelming cultural bias of the developmental psychology database (Fonagy, Luyten, Allison, \& Campbell, 2017). The western, educated, industrialised rich and democratic (WEIRD) countries that account for $12 \%$ of the global population, account for $96 \%$ of the 
Supporting the social triad

participants in psychological samples (Henrich, Heine, \& Norenzayan, 2010). A randomly selected North American undergraduate is more than four thousand times more likely to be a research participant than a randomly selected individual from outside of the WEIRD world. Does this matter from the perspective of mentalizing? Critics of attachment theory would maintain it does (Keller, 2018). Indeed, the definition of sensitive responsiveness used in our work (cited by Aival et al.) and others is based on face-to-face interaction with the caregiver where the infant's point of view is paramount. Both in the verbal and the non-verbal exchange, the serve-and-return description of caregiver-child interaction dominates; the child can serve and the sensitive other returns in a contingent, often cross-modal response. The face-to-face interaction with the caregiver prototypically serves to enable the infant and child to learn about themselves as an independent agent. As we have suggested, only secondarily do they learn about the other through the identification of common assumed elements between intentional states of other and the self (the simulation theory of theory of mind).

From all that we know (and we know all too little, see above) about caregiving in the non-WEIRD world, the physically proximal aspect of the attachment relationship may still be omnipresent, but the infant is more commonly cared for by multiple caregivers in a caregiving network; the infants are physically held but they tend to be facing outward, learning to see the world as others see it. The caregiver's role is to support the process of learning about others' perspectives. The infant is, as it were, an apprentice: guided, directed and instructed to be a social learner. The process is both explicit and implicit, but the emphasis is on the other and only secondarily about the self. In this context, 
Supporting the social triad

attachment, narrowly interpreted as to an individual parent and focused on that dyadic relationship, is of limited survival value.

The shift in status of the infant (from emperor to apprentice) provides a framework for interpreting some of the findings from Aival et al.'s systematic review. Participants from individualistic cultures may show an earlier development and better accuracy in theory of mind because of variability in the weight given to the importance with which the differences between self and others' beliefs are regarded. The dyadic turn taking and smoothly completed interactions of western mothers and infants arguably sensitize infants to use personal qualities and cognitive attributes as the primary reference of action. In such interaction, the infant looks for and alights upon the caregiver's representation of them as the central agent. But being accurately re-presented (mentalized) is only one way that the social trust in the other as a source of reliable knowledge can be generated. In a socializing environment where children and caregivers engage in multiple simultaneous activities, it is preferable, from an evolutionary perspective, for the infant to be sensitized to attend to others' wishes and interests, and to focus on these as the primary reference of actions. A behavior is appropriate if it fits with ongoing collaborative activities. Confidence in relation to learning comes not just from feeling recognised as a self by another, but from feeling the self as aligned with a broader social entity (i.e., a community).

This may go some way to explaining Aival et al.'s observation that collectivistic cultures appear to manifest more empathic concern than individualistic cultures. Participants from individualistic cultures, in perspectivetaking tasks, appear more attuned to self-other difference, while those from 
Supporting the social triad

collectivistic cultures appear in general to be more accurate in interpreting others' feelings. Further, the observation that collectivistic participants manifest greater distress on observing another's suffering is in line with the assumption that the nature of early developmental experience in individualistic culture leads to a bias towards egocentricity whereas collectivist culture introduces a bias towards the apperception of others' experience.

So where does this kind of reasoning lead the relationship between the social learning triad of attachment, mentalizing and epistemic trust? Perhaps, biased by our research paradigms, we have been in our past writings overly focused on the importance of the nuclear family in predicting vulnerability relative to the wider social context that may underpin the experience of trust or mistrust. What Aival et al.'s timely review draws our attention to is the potential wider social causes of trust and mistrust. Coming from an attachment theory background we have emphasised the individual's experience of being inadequately mentalized as the root cause of an incapacity to trust. This may have been inappropriate and was probably the consequence of our own cultural bias. From a broader social perspective, the cultural context in which nuclear families exist - the neighbourhood, the school, the community, the "background level" of social sensitivity - may be as or more important in establishing an environment to create epistemic trust adequate for social learning. Trust in knowledge may equally depend on the individual's experience of fitting in their experience of the broader social network. Perhaps what we need, as Aival et al.'s paper on the cultural dimensions of mentalizing draws attention to, is a kind of Copernican revolution in developmental science that comes to regard the family not as a closed system but as embedded in a community. 
Supporting the social triad

Aival et al. demonstrated cultural differences in relation to the role of mentalizing in psychological disorder, with bodily symptoms more pervasive in samples from collectivistic cultures. Perhaps differences in patterns of mental disorder speak to the priority or value a society places on agency embodied in observable and experienced action supported by the coordination of an entire community versus agency nested in self-recognition, in turn dependent on the recognition of self-other difference in perspective. Aival et al. have, in this wonderfully interesting, thoughtful and imaginative paper, opened up a rich topic. Within the question about the role of culture in mentalizing, there are a whole series of other questions about the relationship between how mind and body come to be embodied in a sense of agency, and how we come to see ourselves as both belonging to a social group and yet valuing our individual identity.

\section{Funding acknowledgement}

The corresponding author is in receipt of a National Institute for Health Research (NIHR) Senior Investigator Award (NF-SI-0514-10157), and was in part supported by the NIHR Collaboration for Leadership in Applied Health Research and Care (CLAHRC) North Thames at Barts Health NHS Trust. The views expressed are those of the authors and not necessarily those of the NHS, the NIHR or the Department of Health and Social Care.

\section{References}

Aival, E., Rothschild-Yakar, L., \& Kurman, J. (2019). Keeping Culture in Mind: A Systematic Review and Initial Conceptualization of Mentalizing from a 
Supporting the social triad

Cross-Cultural Perspective. Journal Clinical Psychology: Science and Practice.

Fonagy, P., Luyten, P., Allison, E., \& Campbell, C. (2017). What we have changed our minds about: Part 2. Borderline personality disorder, epistemic trust and the developmental significance of social communication. Borderline Personality Disorder and Emotion Dysregulation, 4, 9. doi: 10.1186/s40479-017-0062-8

Henrich, J., Heine, S. J., \& Norenzayan, A. (2010). The weirdest people in the world? Behavioral and Brain Sciences, 33(2-3), 61-83. doi: 10.1017/S0140525X0999152X

Kahneman, D. (2011). Thinking, fast and slow. New York, NY: Farrar, Strauss and Giroux.

Keller, H. (2018). Universality claim of attachment theory: Children's socioemotional development across cultures. Proceedings of the National Academy of Sciences, 115(45), 11414-11419. doi:

10.1073/pnas.1720325115 